\title{
A metanephric adenoma of the kidney associated with polycythemia: A case report
}

\author{
PENGFEI WANG, YUAN TIAN, YUEHAI XIAO, YANG ZHANG, FA SUN and KAIFA TANG \\ Department of Urology, Affiliated Hospital of Guizhou Medical University, Guiyang, Guizhou 550004, P.R. China
}

Received January 11, 2015; Accepted September 24, 2015

DOI: $10.3892 / \mathrm{ol} .2015 .3868$

\begin{abstract}
Metanephric adenoma (MA) of the kidney is a rare and frequently benign tumor with a favorable prognosis that is often diagnosed following surgical treatment. In the present study, a 54-year-old female patient presented with complaints of intermittent right-flank pain and anterior abdominal pain occurring over 2 years and sporadic gross hematuria occurring over 3 months. Ultrasonography and computerized tomography imaging revealed a neoplasm lesion localized in the right kidney. Successful open approach radical nephrectomy was performed and post-surgical histopathological examination verified the lesion as a MA of the kidney. Radical nephrectomy, cryoablation or radiofrequency may used to treat MA and a selective panel of immunostains, including WT1, EMA and AMACR, may be useful for diagnosis.
\end{abstract}

\section{Introduction}

Metanephric adenoma (MA) is a rare and frequently benign tumor that accounts for $0.2-0.7 \%$ of adult renal epithelial neoplasms $(1,2)$. MA is observed predominantly in women, with a 2:1 female to male ratio (3). Currently, $<200$ cases have been reported in the literature, often through case reports. The clinical presentation of MA is similar to malignant renal masses; MA possesses two distinct renal lesions, which share several morphological and immunohistochemical features with solid variants of papillary renal cell carcinomas (2). Consequently, this may lead to potential misdiagnosis and inadequate treatment. The present study reports the case of a

Correspondence to: Dr Kaifa Tang or Dr Fa Sun, Department of Urology, Affiliated Hospital of Guizhou Medical University, 9 Beijing Road, Guiyang, Guizhou 550004, P.R. China

E-mail: doc.tangkf@hotmail.com

E-mail: sfguizhou123@163.com

Abbreviations: CT, computed tomography; MA, metanephric adenoma

Key words: metanephric adenoma, polycythemia, immunohistochemical, papillary renal cell carcinoma, radical nephrectomy 54-year-old female that presented with MA associated with polycythemia.

\section{Case Report}

A 54-year-old female patient presented to the Affiliated Hospital of Guizhou Medical University (Guiyang, China), with complaints of intermittent right flank pain and anterior abdominal pain that occurred over a 2-year period and sporadic gross hematuria that occurred over 3 months in March 2013. The patient had no other symptoms. Physical examination revealed no significant conclusions. Urinalysis revealed hematuria in the urine culture and a routine blood examination exhibited a hematocrit (Hct) volume of $61 \%$, a hemoglobin volume of $174 \mathrm{~g} / \mathrm{l}$, and a red blood cell count of $6.2 \times 10^{12}$ cells $/$. Ultrasonography and computerized tomography (CT) imaging revealed a neoplasm lesion localized in the right kidney. No lymphadenopathy was detected (Fig. 1A and B). Following discussion with the patient and the patient's family, a traditional open surgical treatment was proposed. Subsequent to extensive discussion with urologists of the Affiliated Hospital of Guizhou Medical University an open approach radical nephrectomy was performed. Macroscopically, the tumor consisted of renal tissue measuring $8.0 \times 6.0 \times 5.0 \mathrm{~cm}$ in size, and was a well-circumscribed, soft, white-gray mass with a cut surface that was focally friable and accompanied by necrosis (Fig. 1C and D).

A hematoxylin and eosin staining kit (Nanjing Jiancheng Bioengineering Institute, Nanjing, China) was applied to the resected specimen. Microscopic examination (IMT-2; Olympus Corporation, Tokyo, Japan) revealed that the cellular mass was composed of hyperchromatic cells with scant cytoplasm, tightly packed tubules and glomeruloid-like structures (Fig. 2A and B). The tumor cells were stained with antibodies against S-100 protein (polyclonal rabbit anti-human; 1:100; cat. no. BA0120, Vimentin (polyclonal rabbit anti-human; 1:100; cat. no. PB0378), common acute lymphoblastic leukemia antigen (CD10; monclonal mouse anti-human; 1:200; cat. no. BM3410), cytokeratin (CK; polyclonal mouse anti-human; 1:100; cat. no. BA4051), $\alpha$-methylacyl-coenzyme-A racemase (AMACR; monoclonal mouse anti-human; 1:200; cat. no. BM1712), CK7 (monoclonal mouse anti-human; 1:200; cat. no. BM1618), and Wilms' tumor antigen (WT1; monoclonal mouse anti-human; 


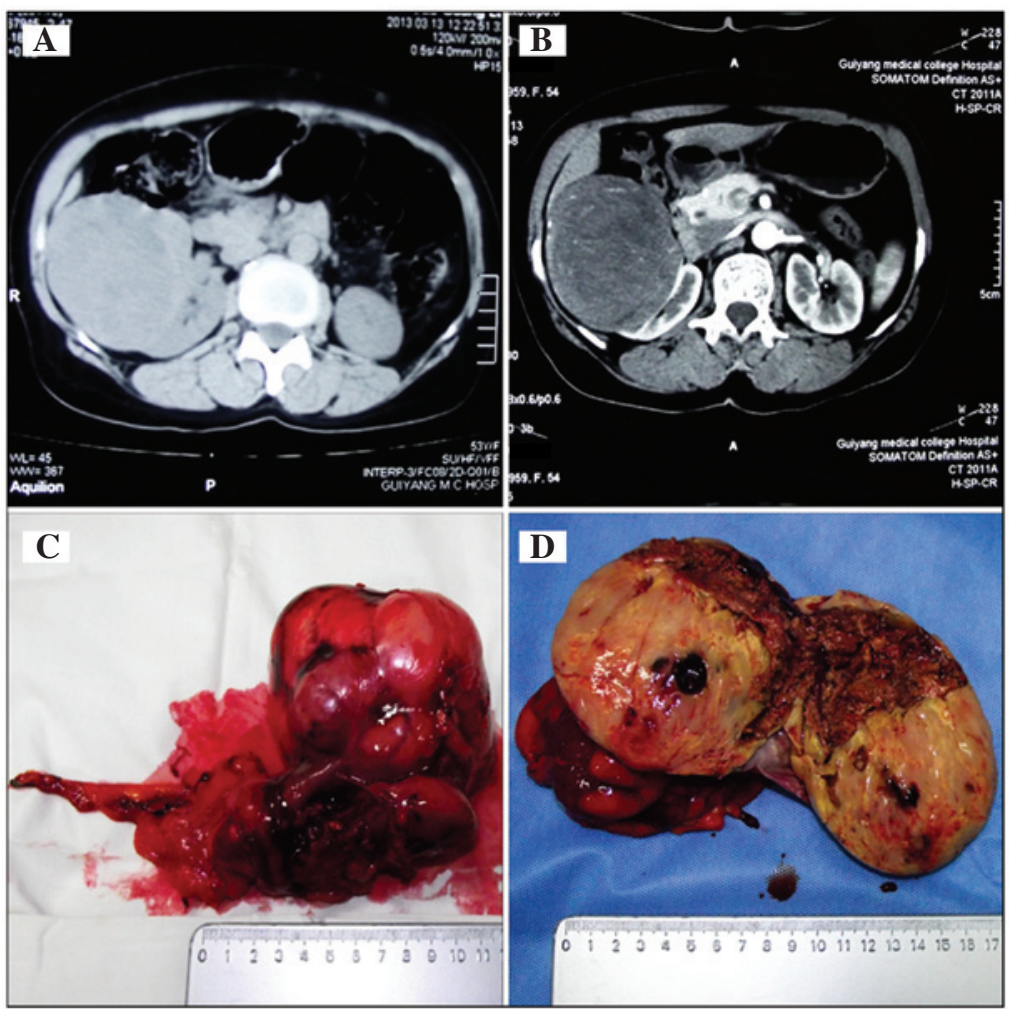

Figure 1. (A) CT scan revealing a neoplasm lesion in the right kidney with no lymphadenopathy. (B) Enhanced CT scan. (C) The tumor following open approach radical nephrectomy. (D) The tumor exhibiting necrosis. CT; computed tomography.

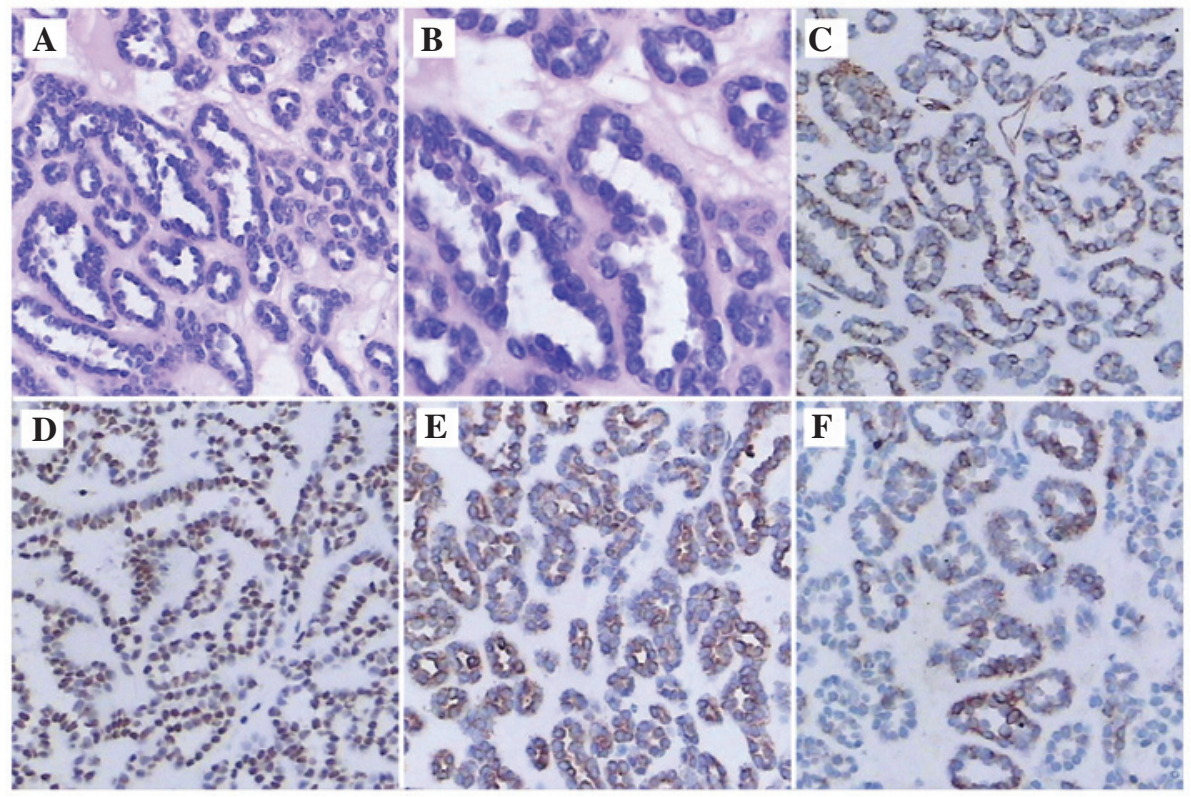

Figure 2. Hematoxylin and eosin staining of the tumor cells. (A and B) Histopathology of the resected specimen (magnification, x200 and x400, respectively). (C-F) Immunohistochemical staining of the tumor cells demonstrating the expression of: (C) Vimentin (magnification, x200); (D) Wilms' tumor protein (magnification, x200); (E) CK (magnification, x200); and (F) CK7 (magnification, x200). CK, cytokeratin.

1:200; cat. no. MK3212) (all purchased from Boster Inc., Wuhan, China). The tumor cells expressed Vimentin, WT1, CK and CK7 (Fig. 2C-F); however, the cells did not express S-100 protein, AMACR or CD10 (data not shown).

The duration of the follow-up was 20 months. Every 6 months, the patient received a routine blood examination and a renal ultrasound. The patient was alive at 20 months, with no clinical complaints. At the first 6-month follow-up the routine blood examination demonstrated a Hct volume of $46 \%$, a hemoglobin volume of $152 \mathrm{~g} / 1$, and a red blood cell count of $5.6 \times 10^{12} \mathrm{cells} / 1$. At the second 6-month follow-up the renal ultrasound revealed there was no metastasis.

Written informed consent was obtained from the patient prior to publication of the case report. 


\section{Discussion}

MA is characterized as a mass consisting of spindle cells associated with epithelial cells (2). In 1988, Mostofi et al (4) described MA as a distinct nosologic entity among renal neoplasms, with tubular-like epithelium cells. MA is a rare renal epithelial neoplasm and has a peak age of occurrence in the fifth or sixth decade of life. It is closely associated with other metanephric neoplasms, including pure stromal lesions and metanephric adenofiromas $(3,5)$. Clinically, MA may present with hematuria, flank pain, hypertension or abdominal mass. In total, $12 \%$ of patients present with polycythemia vera in addition to MA, which is a higher percentage compared with other renal neoplasms $(3,6,7)$.

MA appears as a well-defined, round, solid, soft mass varying between 0.3 and $15.0 \mathrm{~cm}$ in size $(3,8)$. Histologically, MA exhibits uniform small cells with a high nuclear-to-cytoplasmic ratio with an acinar arrangement, without mitosis or embryonic appearance and with a tubular, glomeruloid or papillary structure that is distributed in small round acini, and MA is phenotypically similar to nephroblastomas $(2,3)$. Immunohistochemically, MA expresses CK7 and WT1 and does not express epithelial membrane antigen or AMACR $(2,9)$. To verify the diagnosis of MA, ultrasonography or radiological imaging may be required; the tumors appear hyperechoic with enhanced through-transmission with ultrasonography, while with unenhanced $\mathrm{CT}$ the tumor increases attenuation relative to the adjacent parenchyma (10). However, it remains challenging to differentiate MA from renal cell carcinoma solely by imaging.

Since MA is frequently a benign tumor, it is important to quickly distinguish this lesion from other renal neoplasms, which presents a challenge as MA and renal neoplasms clinically present in an identical manner. Partial or radical nephrectomy is the mainstay of treatment for MA; however, radical nephrectomy may lead to the onset and/or progression of chronic kidney disease, leading to dialysis or transplant treatment. Therefore, open, laparoscopic, robotic or hand assisted partial nephrectomy, or thermoablative procedures are recommended, according to the American Urological Association Guidelines (11). In addition, Conzo et al performed radiofrequency-assisted partial nephrectomy on a patient with MA that led to an excellent hemostasis and a rapid conservative resection, with low morbidity (9).

Overall, MA is an extremely rare benign tumor. Radical nephrectomy, cryoablation or radiofrequency may be used to treat MA. MA cannot be easily distinguished from other malignant neoplasms using imaging alone; however, MA is clearly recognized by microscopy. When diagnosis is challenging, a selective panel of immunostains, including WT1, EMA and AMACR, may be a useful tool.

\section{Acknowledgements}

The present study was supported by the Science and Technology Fund Project of Guizhou Province [grant no. QKHJZ(2013)2051].

\section{References}

1. Amin MB, Amin MB, Tamboli P, Javidan J, Stricker H, de-Peralta Venturina M, Deshpande A and Menon M: Prognostic impact of histologic subtyping of adult renal epithelial neoplasms: An experience of 405 cases. Am J Surg Pathol 26: 281-291, 2002.

2. Mantoan Padilha M, Billis A, Allende D, Zhou M and Magi-Galluzzi C: Metanephric adenoma and solid variant of papillary renal cell carcinoma: Common and distinctive features. Histopathology 62: 941-953, 2013.

3. Davis CJ Jr, Barton JH, Sesterhenn IA and Mostofi FK: Metanephric adenoma. Clinicopathological study of fifty patients. Am J Surg Pathol 19: 1101-1114, 1995.

4. Mostofi FK, Sesterhenn IA and Davis CJ: Benign tumors of the kidney. Prog Clin Biol Res 269: 329-346, 1988.

5. Patel RD, Frederick L, Kohler T and Schwartz B: A case of a metanephric adenoma of the kidney surgically treated with robot-assisted laparoscopic partial nephrectomy. Case Rep Urol 2013: 703859, 2013.

6. Raman SP, Hruban RH and Fishman EK: Beyond renal cell carcinoma: Rare and unusual renal masses. Abdom Imaging 37: 873-884, 2012.

7. Bastide C, Rambeaud JJ, Bach AM and Russo P: Metanephric adenoma of the kidney: Clinical and radiological study of nine cases. Bju Int 103: 1544-1548, 2009.

8. Jones EC, Pins M, Dickersin GR and Young RH: Metanephric adenoma of the kidney. A clinicopathological, immunohistochemical, flow cytometric, cytogenetic, and electron microscopic study of seven cases. Am J Surg Pathol 19: 615-626, 1995.

9. Conzo G, Sciascia V, Palazzo A, Stanzione F, Della Pietra C, Insabato L, Natella V, Radice L and Santini L: Radiofrequency-assisted partial nephrectomy for metanephric adenoma: A case report and literature review. Surg Innov 20: 55-58, 2013.

10. Fielding JR, Visweswaran A, Silverman SG, Granter SR and Renshaw AA: CT and ultrasound features of metanephric adenoma in adults with pathologic correlation. J Comput Assist Tomogr 23: 441-444, 1999.

11. Campbell SC, Novick AC, Belldegrun A, et al: Guideline for management of the clinical T1 renal mass. J Urol 182: 1271-1279, 2009. 